\title{
Time-Bin-Encoded Boson Sampling with a Single-Photon Device
}

\author{
Yu He, ${ }^{1,2}$ X. Ding,,${ }^{1,2}$ Z.-E. Su, ${ }^{1,2}$ H.-L. Huang, ${ }^{1,2}$ J. Qin, ${ }^{1,2}$ C. Wang, ${ }^{1,2}$ S. Unsleber, ${ }^{3}$ C. \\ Chen, ${ }^{1,2}$ H. Wang, ${ }^{1,2}$ Y.-M. He, ${ }^{1,3}$ X.-L. Wang, ${ }^{1,2}$ W.-J. Zhang, ${ }^{4}$ S.-J. Chen, ${ }^{4}$ C. Schneider, ${ }^{3}$ \\ M. Kamp, ${ }^{3}$ L.-X. You, ${ }^{4}$ Z. Wang, ${ }^{4}$ S. Höfling, ${ }^{1,3,5}$ Chao-Yang Lu, ${ }^{1,2}$ and Jian-Wei Pan ${ }^{1,2}$ \\ ${ }^{1}$ Hefei National Laboratory for Physical Sciences at Microscale and Department of Modern Physics, \\ University of Science and Technology of China, Hefei, Anhui 230026, China \\ ${ }^{2}$ CAS-Alibaba Quantum Computing Laboratory, CAS Center for Excellence in Quantum Information and Quantum Physics, \\ University of Science and Technology of China, China \\ ${ }^{3}$ Technische Physik, Physikalisches Instität and Wilhelm Conrad Röntgen-Center for Complex Material Systems, \\ Universitat Würzburg, Am Hubland, D-97074 Wüzburg, Germany \\ ${ }^{4}$ State Key Laboratory of Functional Materials for Informatics, \\ Shanghai Institute of Microsystem and Information Technology (SIMIT), \\ Chinese Academy of Sciences, 865 Changning Rd., Shanghai 200050, China \\ ${ }^{5}$ SUPA, School of Physics and Astronomy, University of St. Andrews, St. Andrews KY16 9SS, United Kingdom
}

(Dated: April 19, 2017)

\begin{abstract}
Boson sampling is a problem strongly believed to be intractable for classical computers, but can be naturally solved on a specialized photonic quantum simulator. Here, we implement the first time-bin-encoded boson sampling using a highly indistinguishable $(\sim 94 \%)$ single-photon source based on a single quantum-dot-micropillar device. The protocol requires only one single-photon source, two detectors and a loop-based interferometer for arbitrary number of photons. The single-photon pulse train is time-bin encoded and deterministically injected into an electrically programmable multi-mode network. The observed three- and four-photon boson sampling rates are $18.8 \mathrm{~Hz}$ and $0.2 \mathrm{~Hz}$ respectively, which are more than 100 times faster than previous experiments based on parametric down-conversion.
\end{abstract}

Boson samping, proposed by Aaronson and Arkhipov [1], has received considerable interest as a model of non-universal (intermediate) quantum computation that can outperform classical computers. It is a sampling task that can be carried out by sending $n$ indistinguishable single photons to a $m$-mode $(m>n)$ optical interferometer, and measure the output photon number distribution. boson sampling doesn't require adaptive measurement, deterministic entangling gates, and makes less stringent demands on device performance than universal optical quantum computing [2].

Previous work [3-7] have demonstrated the working principle of boson sampling using pseudo-single photons produced by spontaneous parametric down-conversion (SPDC) [8]. An intrinsic problem in the SPDC, however, is that the photon pairs are generated probabilistically, and mixed with double pair emission. To overcome this problem, protocols of scattershot boson sampling [9] and spatial/temporal multiplexing $[10,11]$ were proposed to enhance the multi-photon coun$\mathrm{t}$ rate. Yet, so far the boson sampling experiments based on SPDC were limited up to three single-photon Fock states for arbitrary input configurations.

To scale up to a larger number of photons and fast sampling rate, a more efficient route is to use single-photon sources that emit one and only one photon each time [12-14]. To be useful for multi-photon interference, the photons should simultaneously fulfill a checklist that include high system efficiency, near-perfect purity and indistingushability [15]. The singlephoton system efficiency $(\eta)$ can be calculated as the number of single photons output from a single-mode fiber divided by the pulsed laser repetition rate. It would be affected by the quantum efficiency of the emitter, population inversion effi- ciency, extraction efficiency, and loss in the optical path. For $n$-photon boson sampling, the final $n$-fold coincidence rate is proportional to $\eta^{n}$. The photon purity can be characterized by second-order correlation $g^{2}(\tau)$, which gives $g^{2}(0)=0$ for an ideal single-photon source with no multi-photon mixture. The photon indistinguishability can be quantified by the visibility of two-photon Hong-Ou-Mandel interference. High levels of photon purity and indistinguishability are key prerequisites for the boson sampling to be a classically intractable problem [16-18]. Recently, by pulsed $s$-shell resonant excitation [19] of a single quantum dot (QD) embedded in a micropillar, these criteria have been compatibly combined $[15,20,21]$.

For $n$-photon boson sampling, the most straightforward method is to use $n$ separate QDs that emit transform-limited single photons at the same wavelength. This is still challenging due to the inhomogeneity of self-assembled QDs [22] and less perfect two-photon interference from independent QDs [23]. A unique solution is based on only one single-photon emitter, which is more resource efficient. An additional advantage of this approach is that for single photons from the same QD with emission time separated by less than $\sim 1 \mu$ s-shorter than the time scale of spectral wandering - their mutual indistinguishability is protected in a subspace and remains close to unity [20]. Here, we demonstrate boson sampling with a single-photon device, where the photons are time-bin encoded and interfered in an electrically programmable multi-mode network in a loop-based architecture [24-26]. Such a setup also allows us to track the dynamical evolution step-by-step inside the optical circuit.

Using pulsed resonant pumping [19], the QD-micropillar outputs $\sim 24.8 \mathrm{MHz}$ single photons at the end of a single- 
(a)

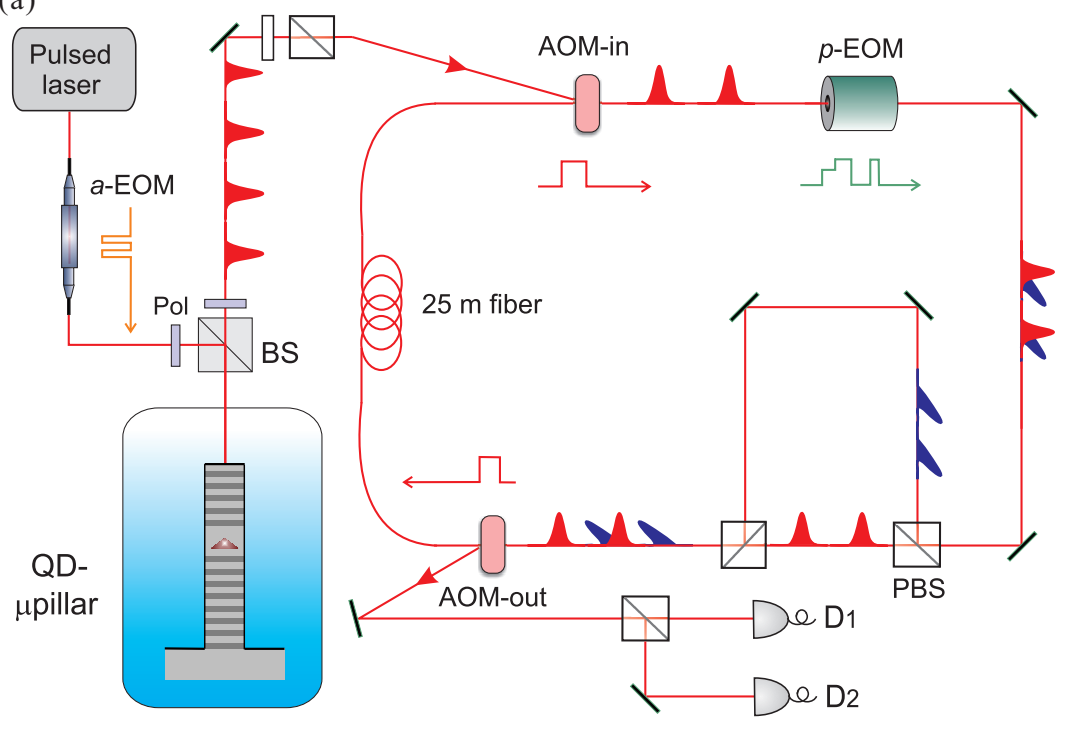

(b)
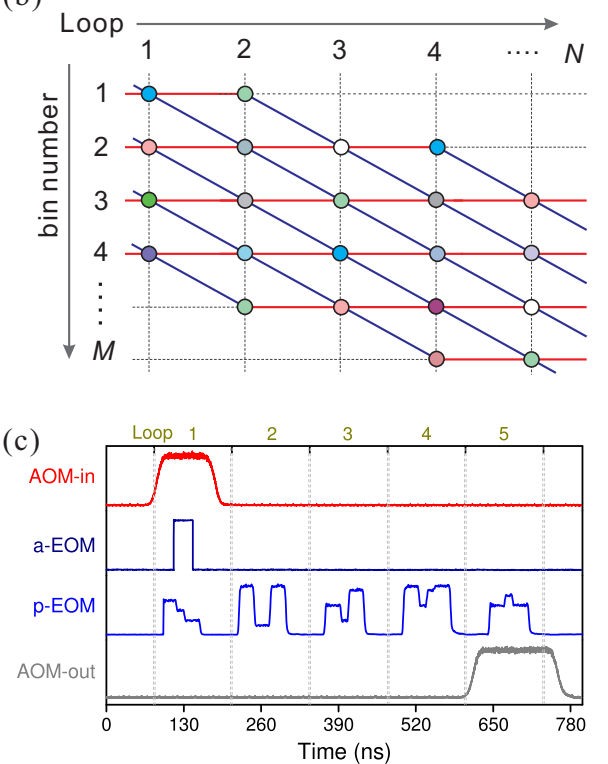

FIG. 1. Boson sampling with a single-photon device. (a) Experimental arrangement. The pumping laser is chopped by a waveguide-based amplitude electro-optic modulator $(a$-EOM) to prepare a single-photon pulse train in designed time bins. The QD is sandwiched between 25.5 lower and 15 upper $\lambda / 4$-thick AlAs/GaAs mirror pairs that form distributed Bragg reflectors, and embedded inside a $2.5 \mu \mathrm{m}$ diameter micropillar. The device is cooled to $7 \mathrm{~K}$ where the QD emission is resonant with the micropillar cavity mode. A confocal microscope is utilized to address the QD, and laser leakage is extingushed by applying cross-polarizers on its both arms. The prepared three or four single photons are injected into a loop by an acousto-optic modulator (AOM). An electro-optical modulator ( $p$-EOM) rotates the polarization controlled by a pulse sequence. The red (blue) pulse in the loop denotes horizontal (vertical) polarization. A $25 \mathrm{~m}$ single-mode fiber is used to increase the loop length to $130 \mathrm{~ns}$ (10 bins). After several loops of evolution (see text for details), the photons are ejected out of the loop by the AOM-out and detected with two superconducting nanowire single-photon detectors. (b) An equivalent beam splitter network unravelling the dynamics of $M$ time bins circulating for $N$ loops. The circles denote beam splitter operations and their color coding represents arbitrary, electrically programmable coupling ratios. The red and blue line evolution represents the trajectory for horizontal and vertical polarization, respectively. (c) Electrical pulse sequences for implementing Boson sampling. The whole system is time synchronized to the pulsed laser.

mode fiber, of which $12.9 \mathrm{MHz}$ are eventually detected by a superconducting nanowire single-photon detectors with an efficiency of $52 \%$ and a dead time of less than $13 \mathrm{~ns}$ (see Fig. 1(a) and Supplemental Materials [27] for more details). Such a single-photon source is $\sim 10$ times more efficient than the state-of-the-art SPDC [28]. Second-order correlation of $0.027(1)$ is observed at zero time delay, proving the highly purified single-photon Fock state. Thanks to the $s$-shell resonant excitation that eliminates dephasings and emission time jitter, we observe two-photon interference visibilities of 0.939(3) for two photons with their emission time separated by $13 \mathrm{~ns}$.

To implement the multi-mode interferometer, we utilize a scalable time-bin encoding scheme [24-26], as illustrated in Fig. 1(a). For each experimental period, $M$ time bins (each loaded with one or zero photon) are injected into a loop by an acousto-optical mudulator (AOM) and circulated for $N$ loops. Such a loop-based architecture is equivalent to an $M$-mode beam splitter network with a depth of $N$, as illustrated in Fig. 1(b). Here, the polarization degree of freedom acts as the spatial mode in the conventional boson sampling model. The beam splitter operations, denoted by the circles in Fig. 1(b), are effectively realized using a polarization-rotation electrooptic modulator ( $p$-EOM) with dynamically programmable coupling ratio. After the $p$-EOM, a polarization-dependent asymmetric Mach-Zehnder interferometer delays the vertical polarization for one time-bin length ( $\sim 13 \mathrm{~ns})$, regarding to the horizontal polarization, which realizes the displacement operation of the time bins.

After $N$ loops of evolution, the $M$ bins are ejected out of the loop by another AOM, and the output distribution are obtained by registering all the single-photon detection events in real time and postprocessing [27]. In this experimen$\mathrm{t}$, the optical transmission efficiency per loop is $83.4 \%$, and the injection and extraction efficiency in each AOM is $85 \%$. We calibrate the mode matching of the time-bin loop using Mach-Zehnder interference, and we obtain an interference visibility of $99.5 \%$. The time-bin encoding scheme naturally complements the single-photon pulse train. We note that the loop-based architecture is intrinsically stable, electrically programmable, and resource efficient. For an arbitrary $n$ boson sampling, this scheme requires only one single-photon emitter, two optical switches, two EOMs, and two fast detectors, whereas the traditional spatial-encoding approach requires $n-1$ optical switches, $\sim n^{4}$ beam splitters, and $\sim n^{2}$ detectors [27]. Furthermore, it relaxes the overhead of actively demultiplexing a single photon source [29] or building an array of multiple identical single photon sources [22].

We implement boson sampling with $n=3$ and $n=4$ input 
(a)

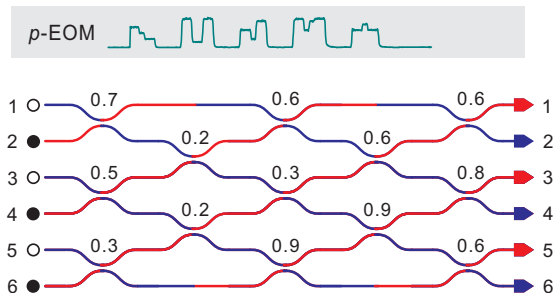

(b)

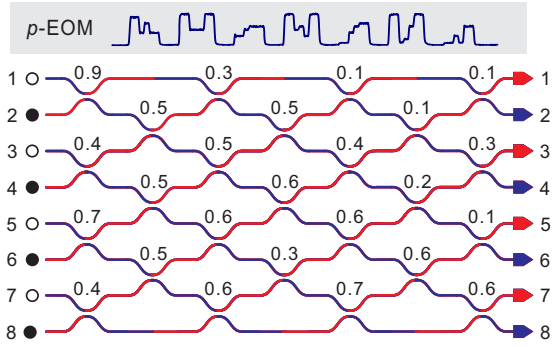

(c)

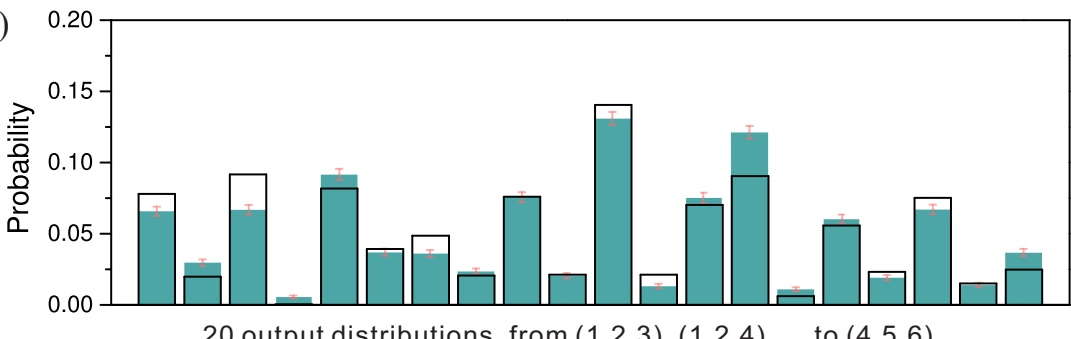

(d)

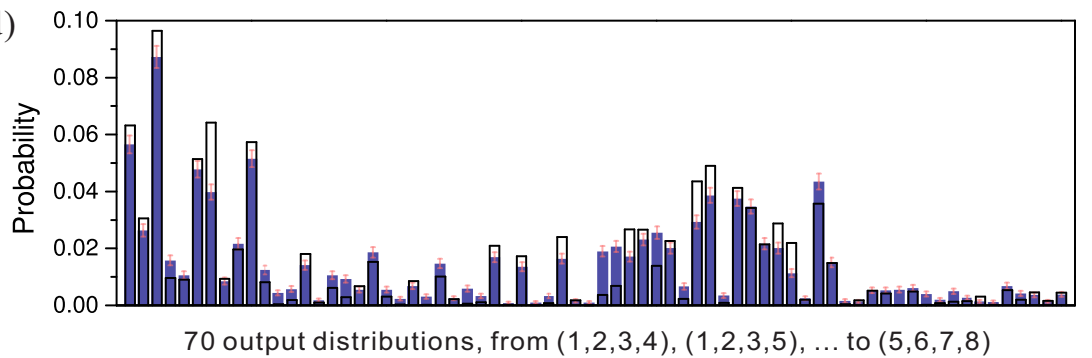

FIG. 2. Experimental results for 3- and 4-boson sampling. (a), (b) The equivalent 3- and 4-boson sampling circuits implemented. The upper panel shows the electrical pulse sequence that drives the $p$-EOM and programs the circuits. The inputs to the circuits are one (zero) photon Fock state, represented by solid (empty) circles. (c), (d) The measured relative frequencies of various output combinations, denoted by $(i, j, k$ ) where $i, j$, and $k$ are the output modes as labelled in (a) and (b). The solid bars indicates the normalized coincidence rate for different output distribution. The empty bars are theoretical calculations in the ideal case. The error bar represents one standard deviation from Poissonian counting statistics.

(a)

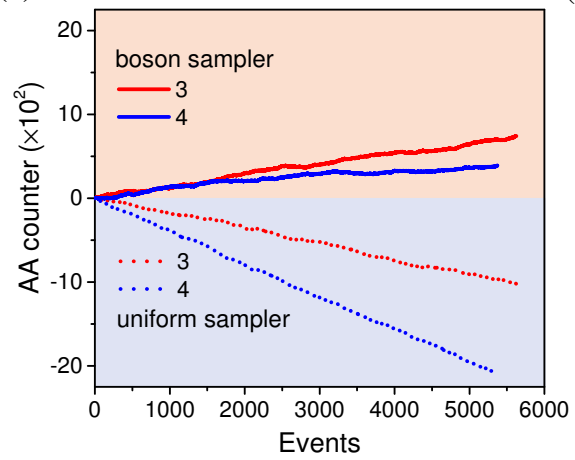

(b)

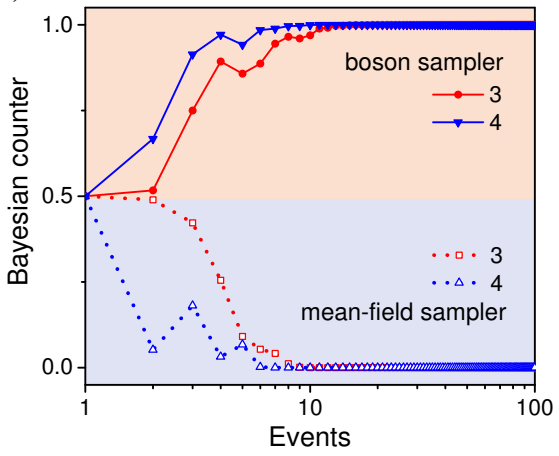

(c)

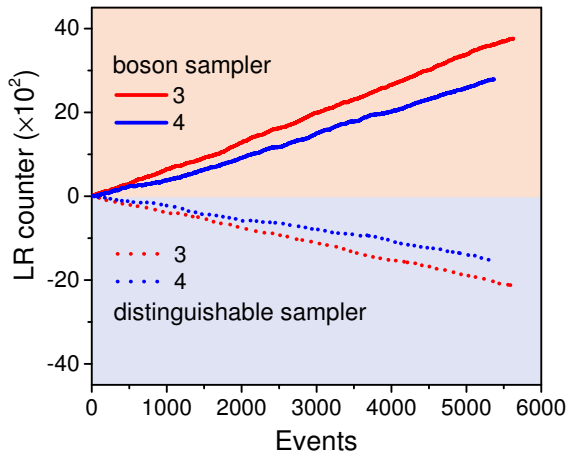

FIG. 3. Validating boson sampling results. Solid lines are tests applied on the experimental data. Dotted lines are tests applied on simulated data generated from the three alternative hypotheses. The counter is updated every single event and a positive value indicates the data is obtained from a genuine boson sampler. Red lines are results for 3 bosons and blue lines are for 4 bosons. (a) Using the Aaronson and Arkhipov (AA) test to rule out the uniform distribution. (b) The Bayesian analysis is applied to ditinguish from mean-field distribution. (c) Standard liklihood ratio test is applied to discriminate the data from a distinguishable sampler.

photons, propagating them through the loop-based interferometers with $m=6$ and $m=8$ modes for $N=5$ and $N=7$ loops, respectively. Figure 2(a) and 2(b) show two typical equivalent boson sampling circuits, programmed by electric pulse sequences shown in the upper panels that drive the $p$-EOM [27]. We measure no-collision (one photon per output-mode) coincidence events of 20 and 70 different combinations of output distributions for the 3- and 4-boson sampling, respectively. A total of 5626 and 5372 events are recorded in the 3and 4-boson sampling within 5 minutes and 7.6 hours, respectively. The high-efficiency single-photon source allows us to run the 3-boson sampling $\sim 100$ times faster than the best pre- vious work with SPDC [3-7]. The experimental probability distributions (solid bar) are shown in Fig. 2(c)-(d) with theoretically evaluated distributions (empty bars) through caculating the permanent of the corresponding submatrix. To make a comparsion between the normalized distributions obtained experimentally $\left(q_{i}\right)$ and theoretically $\left(p_{i}\right)$, the measure of the fidelity: $F=\sum_{i} \sqrt{p_{i} q_{i}}$ is applied. From the data shown in Fig. 2(c)-(d), we obtain a fidelity of 0.993(2) for the 3-boson sampling, and 0.973(6) for the 4-boson sampling, respectively. The slight fidelity decrease might be mainly due to the mode mismatching $(0.5 \%)$ per loop, where the 3 - and 4-boson sampling runs for 5 and 7 loops, respectively [30]. 

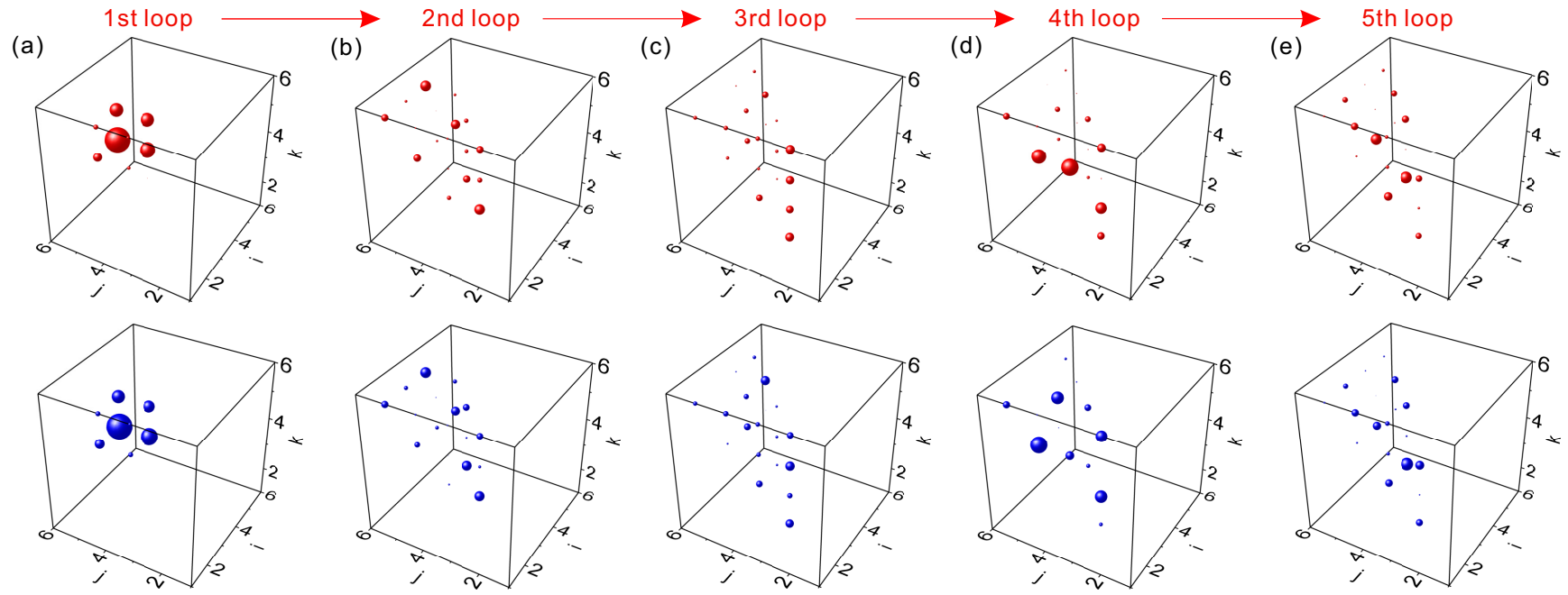

FIG. 4. Tracking boson sampling dynamics. The output distribution is measured after 1 (a), 2 (b), 3 (c), 4 (d), and 5 (e) loops evolution in the 3-boson sampling circuit shown in Fig. 2(a). The probability of finding three photons in the output-mode distributions $(i, j, k)$ (see Fig. 2 caption) are plotted using a sphere centered at coordinates $(i, j, k)$, where the volume of the sphere is proportional to the occurring frequency. The measured fidelities from the 1st to the 5th loop are 0.981(6), 0.985(0), 0.991(2), 0.967(2), and 0.993(2), respectively. The upper and lower panels are the experimental data and theoretical calculation, respectively.

As the boson sampling is related to a problem strongly believed to be in the \#P-complete complexity class, not only the calculation but also a full certification of its outcome could become exponentially intractable for classical computation. To this end, there have been proposals [31-33] and demonstrations $[34,35]$ for validation of boson sampling, and discriminate the genuie boson sampling result with some other type$\mathrm{s}$ of sampling hypothesis. Firstly, we apply the experimental data to the Aaronson and Arkhipov test [31], designed to distinguish the outcome of fixed-input boson sampling from a uniform distribution (see Fig. 3(a)). In our test, the uniform distribution can be conclusively ruled out with $\sim 200$ events. Secondly, we employ the Bayesian analysis [32] to exclude the possibilities of a mean-field sampler [36] (see Fig. 3(b)). With only $\sim 15$ events, a confidence level of $99.8 \%$ is achieved, proving that the output distribution data are from a genuine boson sampler. Finally, we adapt the standard likelihood ratio test $[33,35]$ to rule out the hypothesis that the data could be reproduced with a sampler with distinguishable bosons. Fig. 3(c) shows an increasing discrepancy between indistingushable photons (solid lines) and distingushable photons (dotted lines) against the increase of sampling events, and confirms that our data are indeed expected from highly indistinguishable single photons.

The flexible loop-based architecture in this experiment further allow us to track the dynamical multi-photon evolution in the circuit at intermediate time. Controlled by the ejection time of the AOM-out (Fig. 1a), the output distribution can be measured and monitored, on a loop-by-loop basis. The evolution of the multi-photon scattering of a new 3-boson sampling circuit (supplementary information) at the end of the 1-5 loop are shown in Fig. 4(a)-(e), respectively. The measured fidelities (upper panels) from the 1st to the 5th loop are 0.981(6),
0.985(0), 0.991(2), 0.967(2), and 0.993(2), respectively, in a good agreement with the theoretical calculations (lower panels). Our experiment opens a new way to study multi-particle high-dimensional quantum walks with single quantum emitters $[26,37,38]$. We also anticipate that our platform would be useful for other applications of boson sampling, such as sub-shotnoise quantum metrology [39].

The overall efficiency of the current experiment is mainly limited by the system efficiency of the single-photon source ( $\sim 24.7 \%$, due to losses in light extraction, cross polarization, optical path transmission, and fiber coupling), transmission of the loop-based interferometer ( $\sim 83.4 \%$ per loop), and single-photon detection efficiency $(\sim 52 \%)$. With on-going technological advances on deterministic QD-micropillar [40], background-free laser excitation (to avoid cross-polarization) [41], and high-efficiency superconducting nanowire singlephoton detection [42, 43], boson sampling with rapidly increasing number of photons can be expected. Our work opens up a new avenue to multi-photon quantum computation with single quantum emitters, and brings boson sampling closer to an experimental regime approaching quantum supremacy.

After the first version of our experiment was complete [44], we became aware of a related work on 3-boson sampling using passively demultiplexed single-photon source from a nonresonantly pumped QD [45].

[1] S. Aaronson and A. Arkhipov, in Proceedings of the 43rd Annual ACM Symposium on Theory of Computing (eds Fortnow, L.\& Vadhan, S.), 333 (ACM Press, 2011).

[2] P. Kok, W. J. Munro, K. Nemoto, T. C. Ralph, J. P. Dowling, and G. J. Milburn, Rev. Mod. Phys. 79, 135 (2007); 
[3] M. A. Broome, A. Fedrizzi, S. Rahimi-Keshari, J. Dove, S. Aaronson, T. C. Ralph, and A. G. White, Science 339, 794 (2013).

[4] J. B. Spring et al., Science 339, 798 (2013).

[5] M. Tillmann, B. Dakić, R. Heilmann, S. Nolte, A. Szameit, and P. Walther, Nat. Photon. 7, 540 (2013).

[6] A. Crespi et al., Nat. Photon. 7, 545 (2013).

[7] M. Bentivegna et al. Science Advances 1, e1400255 (2015).

[8] P. G. Kwait, K. Mattle, H. Weinfurter, A. Zeilinger, A. V. Sergienko, and Y. Shih, Phys. Rev. Lett. 75, 4337 (1995).

[9] A. P. Lund, A. Laing, S. Rahimi-Keshari, T. Rudolph, J. L. O’Brien, T. C. Ralph, Phys. Rev. Lett. 113, 100502 (2014).

[10] T. B. Pittman, B. C. Jacobs, and J. D. Franson, Phys. Rev. A 66, 042303 (2002).

[11] F. Kaneda et al. Optica 2, 1010 (2015). C. Xiong et al, Nat. Comm. 7, 10853 (2016).

[12] P. Michler, A. Kiraz, C. Becher, W. V. Schoenfeld, P. M. Petroff, L. Zhang, E. Hu, and A. Imamoğlu, Science 290, 2282 (2000).

[13] C. Santori, D. Fattal, J. Vučković, G. S. Solomon, and Y. Yamamoto, Nature 419, 594 (2002).

[14] I. Aharonovich, D. Englund, and M. Toth, Nature Photonics 10, 631 (2016)

[15] X. Ding, Y. He, Z.-C. Duan, N. Gregersen, M.-C. Chen, S. Unsleber, S. Maier, C. Schneider, M. Kamp, S. Höfling, C.-Y. Lu, and J.-W. Pan, Phys. Rev. Lett. 116, 020401 (2016).

[16] M. Tillmann, S.-H. Tan, S. E. Stoeckl, B. C. Sanders, H. de Guise, R. Heilmann, S. Nolte, A. Szameit, and P. Walther, Phys. Rev. X 5, 041015 (2015).

[17] V. S. Shchesnovich, Phys. Rev. A 91, 013844 (2015).

[18] M. C. Tichy, Phys. Rev. A 91, 022316 (2015).

[19] Y.-M. He, Y. He, Y.-J. Wei, D. Wu, M. Atatüre, C. Schneider, S. Höfling, M. Kamp, C.-Y. Lu, and J.-W. Pan, Nat. Nanotech. 8, 213 (2013).

[20] H. Wang et al., Phys. Rev. Lett. 116, 213601 (2016).

[21] J. C. Loredo et al. Optica 3, 433 (2016).

[22] R. B. Patel, A. J. Bennett, I. Farrer, C. A. Nicoll, D. A. Ritchie, and A. J. Shields, Nat. Photonics 4, 632 (2010).

[23] Y. He, Y.-M. He, Y.-J. Wei, X. Jiang, M.-C. Chen, F.-L. Xiong, Y. Zhao, C. Schneider, M. Kamp, S. Höfling, C.-Y. Lu, and J.W. Pan, Phys. Rev. Lett. 111, 237403 (2013).

[24] K. R. Motes, A. Gilchrist, J. P. Dowling, and P. P. Rohde, Phys. Rev. Lett. 113, 120501 (2014).

[25] P. C. Humphreys, B. J. Metcalf, J. B. Spring, M. Moore, X.-M. Jin, M. Barbieri, W. S. Kolthammer, and I. A. Walmsley, Phys. Rev. Lett. 111, 150501 (2013).
[26] A. Schreiber, K. N. Cassemiro, V. Potoček, A. Gábris, P. J. Mosley, E. Andersson, I. Jex, and Ch. Silberhorn, Phys. Rev. Lett. 104, 050502 (2010).

[27] see Supplemental Materials for more details.

[28] X.-L. Wang et al., Phys. Rev. Lett. 117, 210502 (2016).

[29] H. Wang et al. arXiv:1612.06956 (2016); F. Lenzini et al. arXiv:1611.02294 (2016).

[30] K. R. Motes, J. P. Dowling, A. Gilchrist, P. P. Rohde, Phys. Rev. A 92, 052319 (2015)

[31] S. Aaronson and A. Arkhipov, Quant. Inf. Comp. 14, 1383 (2014).

[32] M. Bentivegna et al., Int. J. Quant. Inf. 12, 1560028 (2014).

[33] T. M. Cover and J. A. Thomas, in Elements of Information Theory 2nd edn, (Wiley-Interscience, 2006).

[34] J. Carolan et al., Nat. Photon. 8, 621 (2014).

[35] N. Spagnolo et al. Nat. Photon. 8, 615 (2014).

[36] M. C. Tichy, K. Mayer, A. Buchleitner, and K. Mølmer, Phys. Rev. Lett. 113, 020502 (2014).

[37] A. Peruzzo, et al. Science 329, 1500 (2010).

[38] A. Schreiber, A. Gábris, P. P. Rohde, K. Laiho, M. Štefaňák, V. Potoček, G. Hamilton, I. Jex, and C. Silberhorn, Science 336, 55 (2012).

[39] K. R. Motes, J. P. Olson, E. Rabeaux, J. P. Dowling, S. J. Olson, and P. P. Rohde. Phys. Rev. Lett. 114, 170802 (2015).

[40] S. Unsleber, Y.-M. He, S. Gerhardt, S. Maier, C.-Y. Lu, J.-W. Pan, N. Gregersen, M. Kamp, C. Schneider, and S. Höfling, Opt. Exp. 24, 8539 (2016).

[41] E. B. Flagg, A. Muller, J. W. Robertson, S. Founta, D. G. Deppe, M. Xiao, W. Ma, G. J. Salamo, and C. K. Shih, Nat. Phys. 5, 203 (2009).

[42] W.-J. Zhang et al. IEEE Photon. J. 8, 2 (2016).

[43] I. E. Zadeh, J. W.N. Los, R. B.M. Gourgues, V. Steinmetz, S. M. Dobrovolskiy, V. Zwiller, S. N. Dorenbos, Preprint at http://arxiv.org/abs/1611.02726 (2016).

[44] Y. He et al. arXiv:1603.04127.

[45] J. C. Loredo, M. A. Broome, P. Hilarie, O. Gazzano, I. Sagnes, A. Lemaitre, M. P. Almeida, P. Senellart, A. G. White, Phys. Rev. Lett. 118, 130503 (2017).

Acknowledgement: Y. He and X. Ding contributed equally to this work. This work was supported by the National Natural Science Foundation of China, the Chinese Academy of Sciences, the National Fundamental Research Program, and the State of Bavaria. 